\title{
Research Article \\ Schemes of eStories for Children with Social Communication Difficulties
}

\author{
Noura Al Ghurair (iD and Ghada Alnaqi \\ Department of Systems and Software Development, Kuwait Institute for Scientific Research, Kuwait City, Kuwait \\ Correspondence should be addressed to Noura Al Ghurair; nghurair@gmail.com
}

Received 3 February 2020; Revised 27 June 2020; Accepted 8 July 2020; Published 28 July 2020

Academic Editor: Alessandra Agostini

Copyright (c) 2020 Noura Al Ghurair and Ghada Alnaqi. This is an open access article distributed under the Creative Commons Attribution License, which permits unrestricted use, distribution, and reproduction in any medium, provided the original work is properly cited.

\begin{abstract}
The current paper presents the development and the evaluation of an Arabic application (app) for electronic stories (eStories) that can be used as an assistive tool in the rehabilitation of children with social communication difficulties. The development process involved engineers and researchers and speech and language pathologists (SLPs) from a rehabilitation hospital, who formulated the design methodology of the Arabic app. This process is critical when designing tools for children with communication difficulties since they have a wide range of differences in their abilities. The evaluation of the app from the standpoint of the SLPs at a local school suggests that the app is easy to use, and the involvement of these SLPs helped in developing a better solution. The results of the usability study on twenty-five students showed positive evaluation with an average score that signifies that the app has above-average usability. This paper highlights the importance of the evaluation process in catering the app for successful user experience.
\end{abstract}

\section{Introduction}

Children with social communication difficulties show significant deficits in language with limited social interaction and verbal commenting. These children frequently struggle in social settings due to deficits in initiating and responding to communication. In the USA, the number of children with communication difficulties has increased [1]. This increase presents new challenges for parents, caregivers, and speech and language pathologists (SLPs) of children with social communication difficulties. Language skills develop quickly throughout the preschool years. Assistive apps are flexible and widespread for teaching children with communication difficulties $[2,3]$ and therefore promise innovative interventions adaptable for various treatments.

Effective treatment and intervention will aid many children with communication difficulties to enhance their level of functioning and subsequently their quality of life. An effective teaching method for children with communication difficulties is breaking down an activity into multiple smaller steps and replacing their representative words with pictures
[2]. Another technique, called Social Stories ${ }^{\mathrm{TM}}$ [4], uses a sequence of images with words representing the steps of daily habits along with the script for expected behaviour for the child. Literature shows that stories help improve children's perception of social expectations [5].

We developed an application that contains four stories based on a request from the SLPs at Kuwait physical medical and rehabilitation hospital (KPMRH). This request was valued and encouraged by funding. The number of SLPs per children with communication difficulties is relatively low at the hospital. Therefore, an assistive tool to enhance the work of these SLPs in supporting these children was needed because of the scarcity of Arabic story applications for children with communication difficulties. The assistive tool, developed with the assistance of the SLPs of KPMRH to accommodate the local culture, contains stories that help the children learn skills associated with activities of daily living. We developed the app and evaluated its usability, and the findings are reported hereinafter. It is the objective of this work to validate the design methodology when providing a solution for tablet application for children with social communication difficulties. 


\section{Background}

Children with communication difficulties show a deficit in narrative comprehension, which negatively impacts their social world. The usage of new technologies for people with communication difficulties mostly addresses communication as well as behavioural analysis, self-stimulation, and social interactions. iOS and Android app stores currently provide several inexpensive apps. Old-generation communicators such as GoTalk, Tango, Dynavox, and Activity Pad have been lately substituted by mobile apps that are easy to use, flexible, scalable, adaptable, educational, and affordable. However, considerable efforts for configuration are required for some apps, so usability is an important factor that guides in developing learning tools.

Hirano [6] created a visual system for planning and organizing children's everyday activities and found improvements in communication and increased efficiency for therapists. Hailpern [7] explored the conduct of nonverbal children, using the computer for evaluating attention, engagement, and vocal behaviour. Monibi [8] installed on a smartphone a customizable library of virtual cards for the activities of autistic children. Hetzroni [9] explored the effects of an app on five children with autism, observing improved communication and the possibility of transferring the newly learned skills from the classroom to daily activities.

Nowadays, children's daily lives are dominated by onscreen activities that include watching television and playing computer games on tablets and other devices [10]. Also, there is a considerable increase in the number and availability of electronic storybooks. The mid-1990s witnessed the appearance of the first picture storybook on CD (Just Grandma and $\mathrm{Me}$ ) [11], and this format has become a substitute for traditional paper books for children. The Association of American Publishers [12] has reported that sales of children's electronic books on phones, tablets, and e-readers increased from 7 million US dollars in 2011 to 19.3 million the next year. This evolution from traditional paper books as the principal source of storybook reading to electronic sources marks a change in the way young children are growing up [13]. Parents have been concerned about the potential adverse effects of technological devices on young children, and there is a need to understand how digital stories can support or hinder the development of literacy in young children when most of them have not yet become conventional readers [14].

Electronic storybooks are mostly stimulating because, besides the involvement in reading or listening to a story, these books also provide technical enhancements that render the experience of reading different from that of traditional paper books. Electronic books typically provide a blend of features, such as animated scenarios and characters and background music and sounds that accompany the text. The child can activate these interactive hypermedia elements and animations with a mouse click or screen touch. Hotspots are pictures with links to animation, sounds, or music. These hotspots may be for entertainment but could also be used to provide useful on-demand help. A huge variety of such digital elements can be incorporated into electronic picture storybooks [15-17].

Stories presented on phones and tablets offer an excuse not to print books. The fundamental challenge is to specify when and how children recall information from electronic storybooks. Research on children has shown different effects of electronic stories, and the way the data in the stories is processed. Adding specific information to electronic storybooks enables multimedia learning, especially in children threatened with language or reading difficulties. The stories that contain animated pictures enriched with music and sound can help integrate nonverbal information and expression and thus help store those elements in memory [18]. Even though there are very few well-controlled studies investigating the effectiveness of social stories, the interventions are frequently used as a treatment option for children with communication difficulties, and most reviews report favourable results [19].

\section{Proposed System}

3.1. Development Process. An Arabic application for eStories was developed for children with communication difficulties. The development process aimed at making the stakeholders active participants from the early stages of the design process to ensure the usefulness, usability, and accessibility of the product. This process is crucial when designing tools for children with communication difficulties since they have a wide range of differences in their abilities. Interaction with children with communication difficulties is very intricate because of their deficiency in communication skills. Understanding the needs of the children is furthermore a challenging task because of their difficulties in verbally expressing their needs or in using gestures instead of words [20]. Augusto [21] preferred applying the user-centred intelligent environments in the development process to the creation of a system that includes individuals with special needs into society. This methodology requires stakeholders who are willing to engage and developers who have the capacity to listen.

Users can contribute to the development process in a number of ways, such as interviews, prototype testing, and system evaluation [22]. These approaches allow designers and users to quickly understand the potentials of the app [23]. In this design approach, stakeholders and users are active contributors of ideas and decision-makers instead of merely a source of information or evaluator of the final product $[24,25]$.

We formed an interdisciplinary workgroup: engineers, researchers, and SLPs who worked together on the application. The SLPs of the rehabilitation hospital provided the main requirements and requested that the application be easy to use with no opportunities for children to make mistakes to avoid frustration. Several meetings were held, including the multidisciplinary team to identify the main functionalities of the app, followed by developing the prototype. The SLPs, from the rehabilitation hospital, were heavily involved in this development process, and their input was thoroughly reviewed and incorporated into the design. 
3.2. Design Guidelines. The literature has a set of proposed recommendations and guidelines which were applied when developing the app [21, 26-29]. The proposed solution takes into consideration these guidelines:

(i) Story narration: The narration of the story was added to enhance accessibility for children who lack reading ability or children who prefer storytelling. The narration sound files were recorded in a professional sound studio to ensure the sound's quality. Although iOS supports text-to-speech, developers preferred sound recording to ensure the correct pronunciation of Arabic words. Arabic text-tospeech in iOS is highly influenced by the iOS updates, and its quality does not always match users' acceptance. Only a limited number of sentences (Table 1) needed to be recorded for the narration of both Kuwaiti dialect and formal Arabic.

(ii) Simple and colourful images: As directed by the guidelines of the World Wide Web Consortium (W3C), soft and mild colours were used to create the images. The images were appealing, as an artist created them. No bright colours were used. Images composed of a limited number of items were utilized not to distract users but to ensure focus. In addition, any elements that might stand out too much were avoided.

(iii) Easy to flip-navigate through pages: The user can easily navigate between the pages. Simple and clear navigation was utilized.

(iv) Animation: a limited number of animations were incorporated in the app, and all animations were simple. The use of pop-up elements and distractions was eliminated.

(v) Caption: Caption was set in navy colour on white background. A contrast between font and background was respected. The caption was not set on picture background since this can make reading text difficult [30]. The text box was clearly separated from the rest of the picture with no overlapping transparent images and text. The iOS system font bold 46 was selected by the developers, as the font typeface and size should be clear and easy to read on-screen [31]. The SLPs at the rehabilitation hospital recommended the use of only Arabic caption in text. Kuwaiti dialect is somewhat different from formal Arabic and difficult to write.

(vi) Characters: Friendly characters were used, reflecting the local culture. The main characters of the stories are two young children: a girl (Lulu) and a boy (Hamad). The Kuwait Institute for Scientific Research (KISR) has the copyright of these two characters as they are used to promote science in the young population.

The following recommendations were also concluded from the SLPs at the rehabilitation hospital during the development process: (i) Animation to be activated when the user taps the item to keep the user focused on the narration

(ii) Caption to be set on/off from the settings to accommodate the user's abilities. Personalizing the app is highly valued, as personalization is a crucial element for successful user experience for children with communication difficulties [29]

(iii) Narration to support Kuwaiti dialect as well as formal Arabic

(iv) Background sound to be set on/off to accommodate the user's needs

(v) Images of displayed items per page to be fully shown and not partially illustrated

3.3. Stories Setup. Four stories were drafted by the SLPs of KPMRH covering daily routines and reflecting the local culture lifestyle. These stories were simple, colourful, and easy to comprehend. Electronic versions of these stories (eStories) were implemented in Xcode swift programming language to develop the Arabic iPad application. The app was built based on the requirements and recommendations of the SLPs at the rehabilitation hospital and consisted of the following:

(i) An introductory page with the proposed four stories (Figure 1).

(ii) A settings page for the selection of narration, caption, background sound, and the dialect (Arabic or Kuwaiti). The settings page can be accessed from the introductory page using a small setting button kept on the upper left corner of the page, as this page will only be used by SLPs, parents, or caregivers.

The primary objective of the eStories app is to enhance the vocabulary and to encourage the integration of children with communication difficulties in their surroundings. Four stories were created (Figure 1), and the characteristics of these stories are listed in Table 1 . The eStories were developed to accommodate the local culture and activities, such as visiting the Kuwait towers and celebrating Kuwait national day, as illustrated in Figure 2.

Application developers are tempted to rely on multimedia elements to replace text-based information. However, stories enhanced with a rich multimedia interface, such as hotspots, may result in poor user experience and reading comprehension problems [29]. Grynszpan [32] showed that ASD children had poorer performance on richer multimedia interfaces. In such children, using these features requires task switching, which seems to cause cognitive overload, as in the case of multitasking in general. Modalities that simulate typical children could be confusing for ASD. Therefore, the eStories incorporated a limited number of animations to attract the interest of the child as in dressing up for school (Figures 3) where the animation is playable, upon request, to divert the child's attention. 
TABle 1: Characteristics of stories.

\begin{tabular}{lccc}
\hline Book & Number of pages & Total words in the text & Total number of sentences \\
\hline Hamad Daily Routine & 15 & 73 & 16 \\
Lulu Daily Routine & 15 & 73 & 16 \\
The Weekend & 12 & 104 & 12 \\
Hamad and Lulu Educational Daily Routine & 11 & 129 & 11 \\
\hline
\end{tabular}

\section{Evaluation}

The development of the app significantly affects performance. The basis of a lot of existing applications has no research evidence; therefore, these applications might have limited usability. We approached a local school for children with communication difficulties to conduct the evaluation study on eStories. The support of the school management and the perseverance of the staff was an encouraging factor to conduct the study.

The proposed app was tested and verified by six SLPs of Al-Nibras School based on the input of the students with communication difficulties. The field evaluation was carried out in April-May 2019 with twenty-five students. The SLPs of the school were heavily involved in the evaluation process to assess the feedback of students with communication difficulties on the usability study. The participatory design adopted in the development process is optimized with the usability study reflecting the users' behaviours.

4.1. Participants. Twenty-five students aged 5-12 participated in the evaluation. The attributes of the students are listed in Table 2, and Figure 4 shows the ages of the students. These students included six females and nineteen males. The SLPs were positive towards trying new technologies to support their work practices due to the individualized needs of each student.

\section{Methods}

5.1. Reading Session. For this study, each student completed a 15-minute reading session involving a single electronic story. The sessions were held in a quiet, air-conditioned room with suitable lighting within the school and performed by the SLPs. The readings were of the same title, and the story chosen for the reading session was "The Weekend" as it involved both characters. A brief informal training was given to the SLPs on the use of the app before the start of the study. The students experienced the eStories app in an interactive mode with the guidance of the SLPs. The eStories reading session provided the students with the opportunity to explore the features of the app. After reading each page electronically, the student was given the option to play with hotspots on the page. But the SLPs did not encourage or extend conversations beyond the script to ensure the completion of the story within the time frame.

5.2. User Response. The student's experience was evaluated to measure the subjective user's experience based on a 7 point Likert scale that ranged from 1 to 7 . Table 3 is the questionnaire used to measure the student's response to
eStories as to whether the experience is easy, fun, and enjoyable.

Table 4 is the output of the questionnaire presented in the previous table for the 25 students. The answers reflect the difficulty of the app, the fun in reading the story, and the liking of the experience. A high average value indicates that the story was challenging, the story was fun to read, and the experience was enjoyable. Based on Table 4, it was noted that the story was relatively easy and comprehensible. The story was also somewhat fun to read and, to a better extent, likable.

5.3. Usability Test. The System Usability Scale (SUS) test is an established tool and one of the most widely used in the literature. It helps determine the usability of a system [33]. SUS is free, very simple, and short and has been found curiously robust in numerous studies [34-36]. The participants have to answer ten questions on a scale ranging from 1 , "Strongly disagree," to 5 , "Strongly agree," and the result is a score between 0 and 100. A score above 68 signifies that the usability of the application is above average [37]. It is possible to assess the usability of software and determine its quality through the result of the SUS questionnaire, where learning facility is based on four questions out of the ten questions of the SUS test, efficiency on three questions, storage facility on one question, error minimization on one question, and satisfaction on three questions out of the total ten questions [38].

The Arabic version of the SUS translated by AlGhannam [39] was used in the usability study in addition to the English version. Most SLPs are Arabic speakers and felt more comfortable with the Arabic translation than the original English version of SUS. Figure 5 shows the output of the SUS test for all the participants.

The results of the usability study helped draw interesting conclusions about the proposed system. These results showed a positive evaluation for most of the students. The SUS scores averaging 74 were above 68 signifying that overall the usability of the app is considered above average [37].

5.4. Qualitative Observations. We generated two-word clouds for the responses given for the two open-ended questions regarding the features that were most preferred (question 1) and those that needed improvement (question 2 ) in the app. The results for question 1 (Figure 6) showed that the most favoured features of the app were the attractive colours and beautiful drawings, followed by the characters that reflect the local culture. The Arabic/Kuwaiti dialect was also another favoured feature. The results for question 2 (Figure 7) showed that the primary suggested improvements were to introduce caption and narration on demand on each 


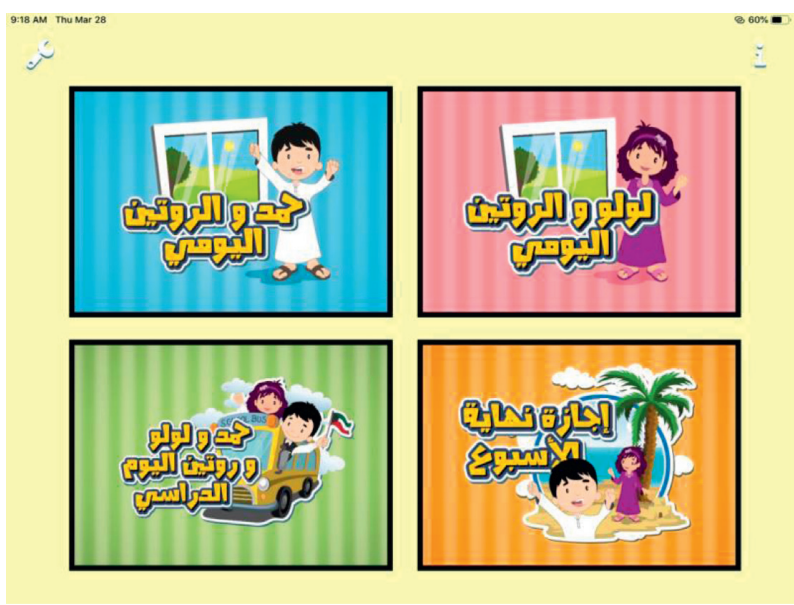

FIgURE 1: eStories.

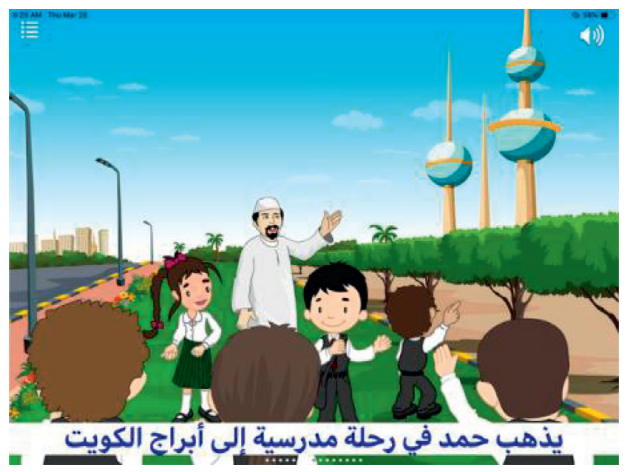

(a)

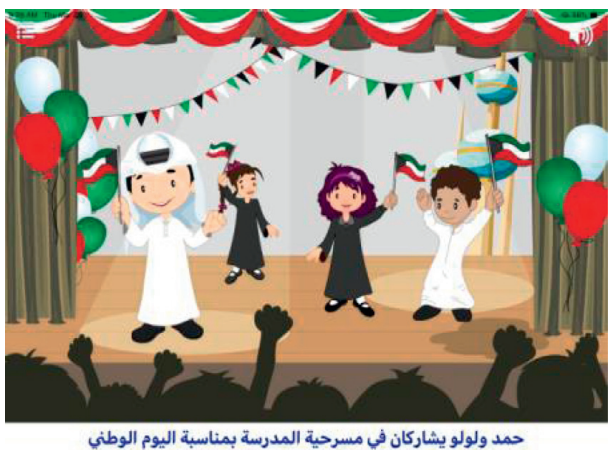

(b)

FIgURE 2: eStories reflecting local culture.

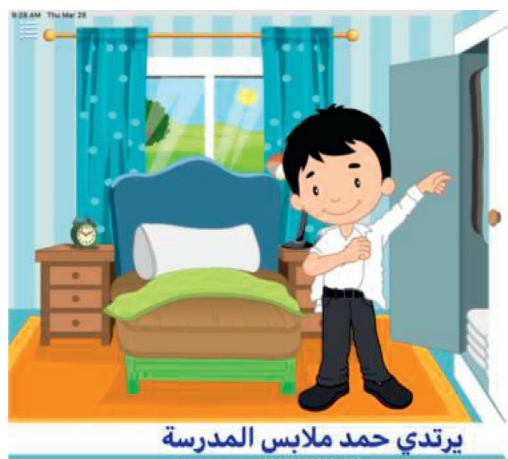

(a)
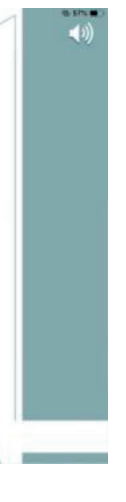

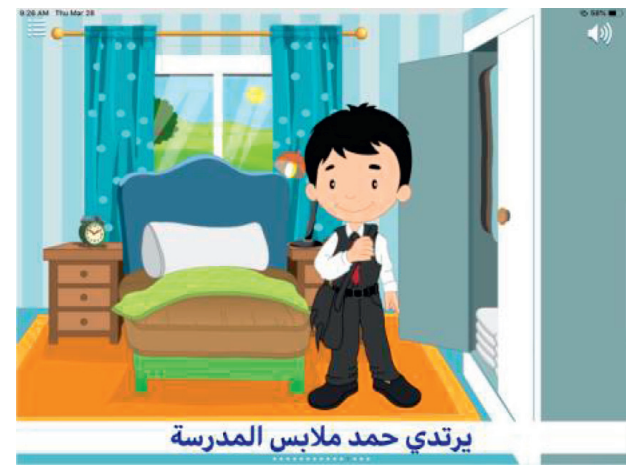

(b)

Figure 3: eStories with minor animation.

page, followed by adding more hotspots to be also used on demand.

\section{Results and Discussion}

A prototype of the eStories application was developed and evaluated in the current study to identify its usability and adequacy for children with communication difficulties.
Information technology can enhance rehabilitation therapy for these children with improvement in socialization, communication, and behaviour [40]. The development process followed the design guidelines presented in the literature and the recommendations of the design team. The evaluation process, on the other hand, was performed on 25 students with communication difficulties accompanied by their SLPs at a local school for special needs. 
TABLE 2: Attributes of students with communication difficulties.

\begin{tabular}{lc}
\hline Attribute & Value \\
\hline Sex & $\mathrm{M} / \mathrm{F}$ \\
Age & $5-12$ \\
Language & Arabic \\
Motor movement & Normal \\
Vision & Normal \\
Hearing & Normal \\
Education level & Primary \\
\hline
\end{tabular}

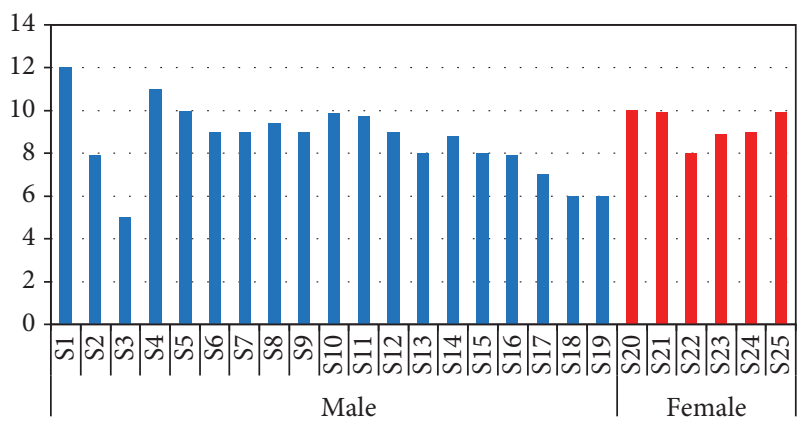

Figure 4: Ages of students.

TABLE 3: Questionnaire to measure users' responses to eStories.

\begin{tabular}{|c|c|c|c|c|c|c|}
\hline \multicolumn{6}{|c|}{ How did you find the story? } & \multirow{2}{*}{$\begin{array}{l}\text { Super hard } \\
\quad 7\end{array}$} \\
\hline 1 & 2 & 3 & 4 & 5 & 6 & \\
\hline \multicolumn{6}{|c|}{ How was your experience with the story? } & Lots of fun \\
\hline 1 & 2 & 3 & 4 & 5 & 6 & 7 \\
\hline \multicolumn{6}{|c|}{$\begin{array}{l}\text { How did you feel about the story? } \\
\text { Don't like the story }\end{array}$} & Best story \\
\hline 1 & 2 & 3 & 4 & 5 & 6 & 7 \\
\hline
\end{tabular}

TABLE 4: Response of a questionnaire to measure users' responses to eStories.

\begin{tabular}{lcc}
\hline Difficult & Fun & Like \\
\hline Average $=1.85$ & Average $=3.81$ & Average $=4.04$ \\
Standard & Standard & Standard \\
deviation $=1.85$ & deviation $=1.02$ & deviation $=1.25$ \\
\hline
\end{tabular}

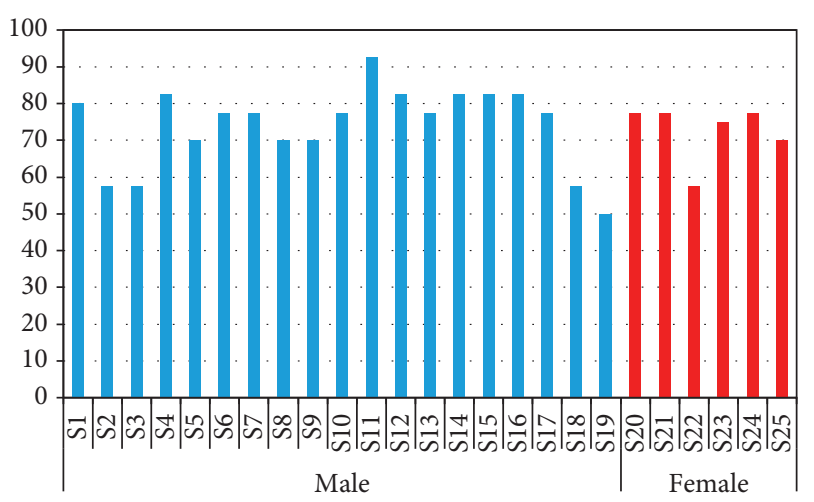

Figure 5: SUS of eStories.

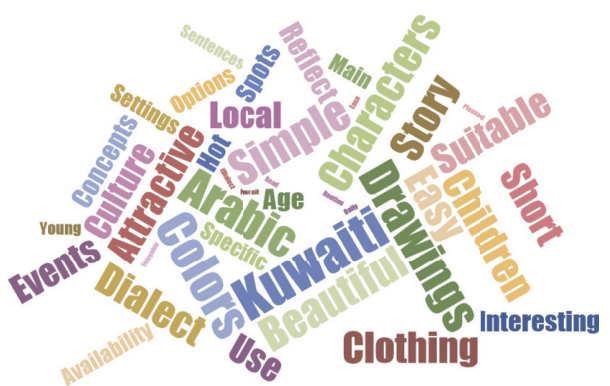

Figure 6: Word cloud representation of responses indicating the most preferred features of the app.

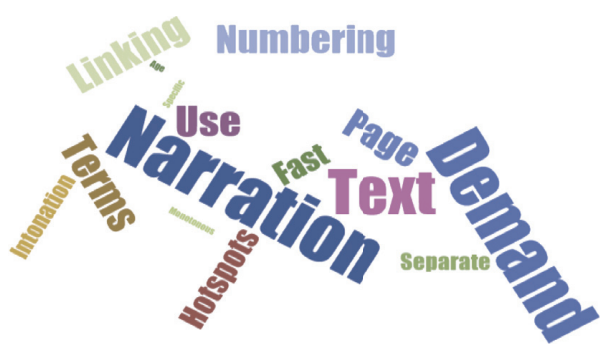

FIGURE 7: Word cloud representation of responses indicating elements in need of improvement in the app.

Both Arabic and English versions of the SUS test were used in the usability study. Each SLP had a preference for using the Arabic or the English version. For future studies, however, the SLPs recommended using a SUS sample test with questions supplied in both English and Arabic concomitantly and not on separate sample tests.

Based on the scores of the SUS test presented in Figure 5, it was noted that a positive evaluation was obtained with a SUS score above average for most of the students. S11 scored highest (92.5) on the SUS test. S11 is a nine-year-and-sevenmonth-old male. His high score at the SUS test is attributed to the questions related to ease of learning. S11, as can be seen in Figure 8, scored highest (100) on the ease of leaning questions. Most of the students scored relatively high on the ease of learning questions which corroborate the observations of the users' responses (Table 4) and the first-word cloud (Figure 6). The students found the story to be relatively easy and comprehensible, and one of the app's preferred features is the fact that it is easy to use. When developing technological apps for people with disabilities, measuring the ease of use is one of the most important aspects [41]. The students, however, averaged 74 on the questions related to satisfaction (Figure 9). This is in line with the responses to the questionnaire to measure the users' responses (Table 4). The story was somewhat fun to read and, to a better extent, likable.

The SUS scores presented in Figure 5 were correlated with the ages of the students found in Figure 4. As can be seen in Figure 10, there is a moderate positive correlation between the age of the student and its SUS score which means that there is a tendency for older students to score higher on the SUS test and vice versa. According to the Pearson correlation coefficient, the value of $R$ was 0.6078 , 


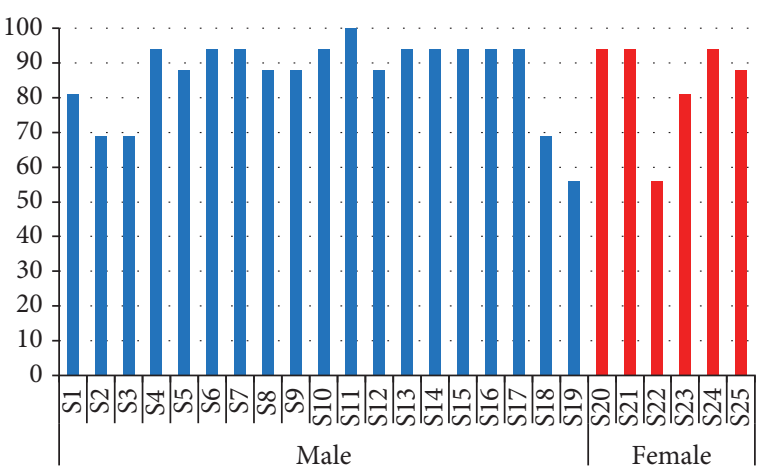

FIgURE 8: Ease of learning.

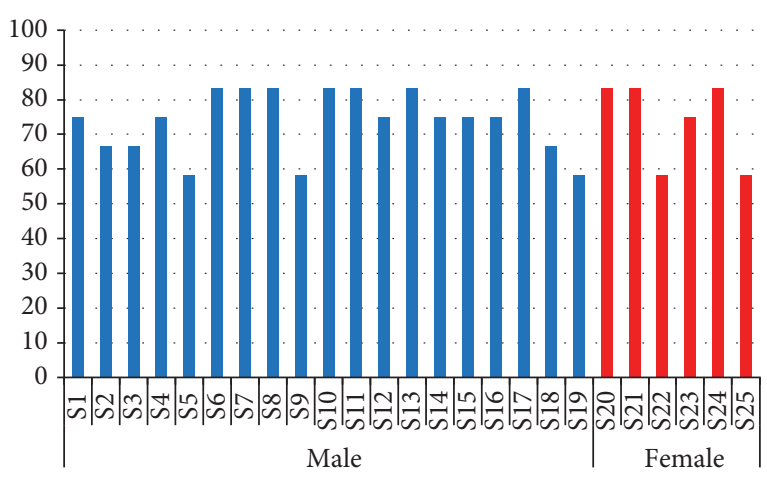

FIGURE 9: Satisfaction.

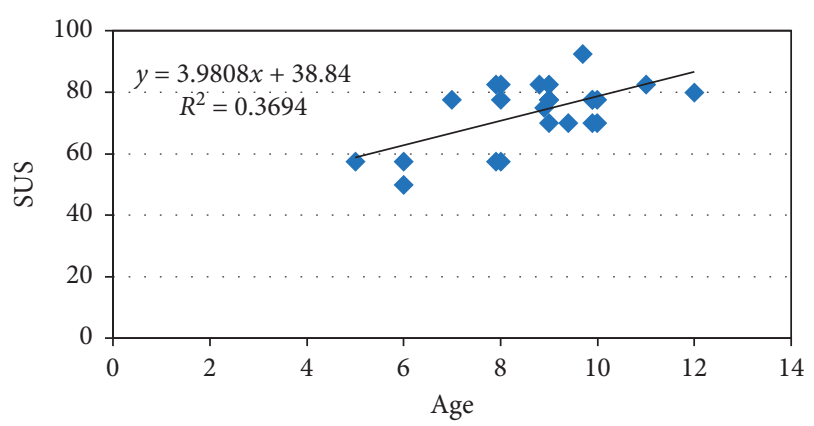

FIGURE 10: Effect of age on SUS.

and the coefficient of determination valued 0.3694 . Younger students below six years old, such as S3, S18, and S19, scored below average on the SUS test. Several SLPs commented that the story is suitable for a specific age group, and this comment is confirmed by the SUS results of students younger than six years old.

It was also noted that the SUS scores were somewhat reflective of the skillsets of the students in using the iPad. S11, who scored highest on the SUS test and the ease of learning questions, has decent communication skills, a reasonable ability to memorize, dependency in self-care, and excellent skills in using the iPad and its settings. He can also make friends and interact with his classmates. S19, on the other hand, who scored lowest on the SUS test (50) and had difficulty in learning (56), is a six-year-old male who performs the activities entrusted to him by verbal guidance. S22 also scored low on the ease of learning questions (56) and on the SUS overall test (57.5). S22 is an eight-year-old female who performs the activities entrusted to her and abides by the rules of the class. She knows how to use the iPad, but she gets distracted upon using it, which explains her low SUS score (57.5).

Overall, the SLPs gave positive feedback on the eStories. The app is reportedly easy to use, and its settings are simple to set with the possibility of showing/hiding caption and narration, turning the background sound on/off, and the availability of dialect selection. The SLPs recommended the introduction of narration on demand for each page. In the settings page, narration can be excluded or included in reading sessions. But it was noted that it was best if narration can be introduced on demand on each page to accommodate the pace of the reading session.

The drawings are simple and colourful. The short story does not cause boredom and reflects the local Kuwaiti/Arabic culture, and its events and characters are familiar to the child. The SLPs suggested adding more hotspots, to be used on demand, to attract the attention of the child when needed even though it was recommended during the design stage of the app to minimize distraction by minimizing hotspots.

According to the SLPs at the local school, the assessment of eStories inspires to think that eStories has the potential to enrich the narration comprehension of children with communication difficulties. The differentiated effects of paper and computer-assisted social stories were studied by Mancil [42] on inappropriate behaviour in children with autism, and the outcomes were slightly better for the computer format than for the paper format. The teachers reported that the computer format was quickly implemented, and the students indicated that they liked the computer-assisted format. Vandermeer [43] concluded that social stories on the iPad proved to be an effective intervention for one of the three children and confirmed that the response might be useful with some children, but not others. Several studies were built on research that supports social stories as a promising practice, and children displayed higher levels of persistence for the electronic story in comparison to the paperback storybook; however, children produced more communicative initiations with a traditional paperback storybook.

\section{Conclusions}

This paper defines the development and the evaluation of the eStories application for children with social communication difficulties, as assistive computing tools are useful in the education of these children. The eStories app follows the design guidelines recommended by the literature [21, 26-29] and the recommendations of the design team. Yet, during the evaluation process, few points were raised that were not obvious in the design process or were planned differently, such as the following:

(i) The introduction of narration on demand on each page, to accommodate the pace of the reading session 
(ii) The addition of more hotspots, to be used on demand to attract the attention of the child during the reading session when he or she loses focus or interest or gets distracted

The evaluation process refined the development procedure by catering the design of the app to accommodate the individualized needs of its users. Personalization is key to successful user experience.

The results of the application evaluation indicate that the app has good acceptability and usability for children with communication difficulties, and there is a real potential for the app to have a positive impact on society by improving interventions and education. eStories represent a way to present a social story to the children and would provide better accessibility to therapy and language stimulation materials for parents to use on the go. Further investigation is recommended into the effect of eStories on reading comprehension, particularly for children of varying ages, abilities, and learning styles. Future collaboration and studies are envisioned where the involvement of other stakeholders, such as psychologists, would be considered for longitudinal studies. The need for collaboration between research bodies and rehabilitation centres or schools should be followed through to support research and development activities and ensure the successful implementation of advanced technologies that enhance the integration of children with social communication difficulties into their society.

\section{Data Availability}

The data used to support the findings of this study are included within the article.

\section{Ethical Approval}

A full explanation was provided to the SLPs involved in the usability study about the details of the research and the roles of the students.

\section{Conflicts of Interest}

The authors declare that there are no conflicts of interest regarding the publication of this paper.

\section{Acknowledgments}

The authors gratefully acknowledge the SLPs of Kuwait Physical Medicine and Rehabilitation Hospital (KPMRH) for supporting the development of the app. The authors would also like to thank and acknowledge the management and SLPs of Al-Nibras School for Special Needs for their help and enormous support in conducting the usability study. Their feedback and input were highly appreciated. The authors salute the SLPs at the Al-Nibras School for their teamwork, commitment, professionalism, and dedication. It was only because of their help that the authors were able to refine the applications to the actual needs of children with communication difficulties. The authors would like to express their sincere gratitude to the Kuwait Foundation for the Advancement of Sciences (KFAS) project number P11613MC-12 for their financial support and the support of the Kuwait Institute for Scientific Research (KISR) on the implementation of this study under the project SD002C.

\section{References}

[1] J. Baio, L. Wiggins, D. L. Christensen et al., "Prevalence of autism spectrum disorder among children aged 8 years-autism and developmental disabilities monitoring network, 11 sites, United States, 2014," MMWR Surveillance Summaries, vol. 67, no. 6, p. 1, 2018.

[2] D. M. Kagohara, L. van der Meer, S. Ramdoss et al., "Using iPods ${ }^{\circledR}$ and iPads ${ }^{\circledR}$ in teaching programs for individuals with developmental disabilities: a systematic review," Research in Developmental Disabilities, vol. 34, no. 1, pp. 147-156, 2013.

[3] L. C. Murdock, J. Ganz, and J. Crittendon, "Use of an iPad play story to increase play dialogue of preschoolers with autism spectrum disorders," Journal of Autism and Developmental Disorders, vol. 43, no. 9, pp. 2174-2189, 2013.

[4] C. A. Gray, "Social stories and comic strip conversations with students with Asperger syndrome and high-functioning autism," in Asperger Syndrome or High-Functioning Autism?, pp. 167-198, Springer, Boston, MA, USA, 1998.

[5] S. Ozdemir, "The effectiveness of social stories on decreasing disruptive behaviors of children with autism: three case studies," Journal of Autism and Developmental Disorders, vol. 38, no. 9, pp. 1689-1696, 2008.

[6] S. H. Hirano, M. T. Yeganyan, G. Marcu et al., "vSked: evaluation of a system to support classroom activities for children with autism," in Proceedings of the SIGCHI Conference on Human Factors in Computing Systems, pp. 16331642, ACM, Atlanta, GA, USA, 2010.

[7] J. Hailpern, K. Karahalios, J. Halle et al., “A3: Hci coding guideline for research using video annotation to assess behavior of nonverbal subjects with computer-based intervention," ACM Transactions on Accessible Computing, vol. 2, no. 2, p. 8, 2009.

[8] M. Monibi and G. R. Hayes, "Mocotos: mobile communications tools for children with special needs," in Proceedings of the 7th International Conference on Interaction Design and Children, pp. 121-124, ACM, Chicago, IL, USA, 2008.

[9] O. E. Hetzroni and J. Tannous, "Effects of a computer-based intervention program on the communicative functions of children with autism," Journal of Autism and Developmental Disorders, vol. 34, no. 2, pp. 95-113, 2004.

[10] V. Rideout, Zero to Eight, Children's Media Use in America, Common Sense Media, San Francisco, CA, USA, 2011.

[11] M. Ito, Engineering Play: A Cultural History of Children's Software, The MIT Press, Cambridge, MA, USA, 2009.

[12] Publishers Weekly, June 21, 2012, http://www.publisherweekly.com/pw/by-topic/childrens/childrens-industry-news/ article/.

[13] C. Burnett, "Technology and literacy in early childhood educational settings: a review of research," Journal of Early Childhood Literacy, vol. 10, no. 3, pp. 247-270, 2010.

[14] E. B. Miller and M. Warschauer, "Young children and e-reading: research to date and questions for the future," Learning, Media and Technology, vol. 39, no. 3, pp. 283-305, 2014.

[15] M. T. De Jong and A. G. Bus, "The efficacy of electronic books in fostering kindergarten children's emergent story understanding," Reading Research Quarterly, vol. 39, no. 4, pp. 378-393, 2004. 
[16] L. Guernsey, M. Levine, C. Chiong et al., Pioneering Literacy in the Digital Wild West: Empowering Parents and Educators, Campaign for Grade-Level Reading, Washington, DC, USA, 2012.

[17] T. A. Zucker, A. K. Moody, and M. C. McKenna, "The effects of electronic books on pre-kindergarten-to-grade 5 students' literacy and language outcomes: a research synthesis," Journal of Educational Computing Research, vol. 40, no. 1, pp. 47-87, 2009.

[18] A. G. Bus, Z. K. Takacs, and C. A. Kegel, "Affordances and limitations of electronic storybooks for young children's emergent literacy," Developmental Review, vol. 35, pp. 79-97, 2015.

[19] S. L. Nichols, S. D. Hupp, J. D. Jewell et al., "Review of social story interventions for children diagnosed with autism spectrum disorders," Journal of Evidence-Based Practices for Schools, vol. 6, no. 1, pp. 90-120, 2005.

[20] M. Z. Aziz, S. A. Abdullah, S. F. Adnan et al., "Educational app for children with Autism Spectrum Disorders (ASDs)," Procedia Computer Science, vol. 42, pp. 70-77, 2014.

[21] J. Augusto, D. Kramer, U. Alegre et al., "The user-centred intelligent environments development process as a guide to co-create smart technology for people with special needs," Universal Access in the Information Society, vol. 17, no. 1, pp. 115-130, 2018.

[22] M. Fabri, P. C. Andrews, and H. K. Pukki, "Using design thinking to engage autistic students in participatory design of an online toolkit to help with transition into higher education," Journal of Assistive Technologies, vol. 10, no. 2, pp. 102-114, 2016.

[23] S. Holmlid, "Participative; co-operative; emancipatory: from participatory design to service design," in Proceedings of the 2009 DeThinking Service; ReThinking Design, Linköping University Electronic Press, Oslo, Norway, pp. 105-118, November 2012.

[24] E. B. N. Sanders and P. J. Stappers, "Co-creation and the new landscapes of design," CoDesign, vol. 4, no. 1, pp. 5-18, 2008.

[25] E. Martin, C. Cupeiro, L. Pizarro et al., "Today I Tell” a comics and story creation app for people with autism spectrum condition," International Journal of Human-Computer Interaction, vol. 35, no. 8, pp. 679-691, 2019.

[26] A. M. Figueroa and R. Juárez-Ramírez, "Developing applications for autistic users: towards an autistic user model,", in Proceedings of the 2013 International Conference on Cloud \& Ubiquitous Computing \& Emerging Technologies, pp. 228-235, IEEE, Pune, India, November 2013.

[27] J. Mintz, C. Branch, C. March et al., "Key factors mediating the use of a mobile technology tool designed to develop social and life skills in children with Autistic Spectrum Disorders," Computers \& Education, vol. 58, no. 1, pp. 53-62, 2012.

[28] J. Mintz, "Additional key factors mediating the use of a mobile technology tool designed to develop social and life skills in children with Autism Spectrum Disorders: evaluation of the 2nd HANDS prototype," Computers \& Education, vol. 63, pp. 17-27, 2013.

[29] N. Pavlov, "User interface for people with autism spectrum disorders," Journal of Software Engineering and Applications, vol. 7, no. 2, p. 128, 2014.

[30] G. Freyhoff, "Make it simple," European Guidelines for the Production of Easy-to-Read Information for People with Learning Difficulties, 1998.

[31] F. AlDhaen, N. El Zant El Kadhi, and H. Al-Obaidy, "Accessible web-based educational system," in Proceedings of the 12th WSEAS International Conference on Neural Networks,
Fuzzy Systems, Evolutionary Computing \& Automation, pp. 42-48, World Scientific and Engineering Academy and Society (WSEAS), Stevens Point, WI, USA, April 2011, http:// www.wseas.us/e-library/conferences/2011/Brasov2/ NEFECIC/NEFECIC-05.pdf.

[32] O. Grynszpan, J. C. Martin, and J. Nadel, "Multimedia interfaces for users with high functioning autism: an empirical investigation," International Journal of Human-Computer Studies, vol. 66, no. 8, pp. 628-639, 2008.

[33] J. Brooke, "SUS-a quick and dirty usability scale," Usability Evaluation in Industry, vol. 189, no. 194, pp. 4-7, 1996.

[34] A. Bangor, P. T. Kortum, and J. T. Miller, "An empirical evaluation of the system usability scale," International Journal of Human-Computer Interaction, vol. 24, no. 6, pp. 574-594, 2008.

[35] A. Bangor, P. Kortum, and J. Miller, "Determining what individual SUS scores mean: adding an adjective rating scale," Journal of Usability Studies, vol. 4, no. 3, pp. 114-123, 2009.

[36] N. Bevan, "International standards for usability should be more widely used," Journal of Usability Studies, vol. 4, no. 3, pp. 106-113, 2009, http://uxpajournal.org/wp-content/ uploads/sites/8/pdf/JUS_Bevan_May2009.pdf.

[37] J. Brooke, "SUS: a retrospective," Journal of Usability Studies, vol. 8, no. 2, pp. 29-40, 2013.

[38] J. M. Tenorio, A. D. Hummel, F. M. Cohrs et al., “Artificial intelligence techniques applied to the development of a decision-support system for diagnosing celiac disease," International Journal of Medical Informatics, vol. 80, no. 11, pp. 793-802, 2011.

[39] B. A. AlGhannam, S. A. Albustan, A. A. Al-Hassan et al., "Towards a standard Arabic system usability scale: psychometric evaluation using communication disorder app," International Journal of Human-Computer Interaction, vol. 34, no. 9, pp. 799-804, 2018.

[40] S. Artoni, L. Bastiani, M. C. Buzzi et al., "Technology-enhanced ABA intervention in children with autism: a pilot study," Universal Access in the Information Society, vol. 17, no. $1,2018$.

[41] M. Dawe, "Desperately seeking simplicity: how young adults with cognitive disabilities and their families adopt assistive technologies," in Proceedings of the SIGCHI conference on Human Factors in computing systems, pp. 1143-1152, ACM, Montreal Quebec, Canada, 2006.

[42] G. R. Mancil, T. Haydon, and P. Whitby, "Differentiated effects of paper and computer-assisted Social Stories ${ }^{\mathrm{TM}}$ on inappropriate behavior in children with autism," Focus on Autism and Other Developmental Disabilities, vol. 24, no. 4, pp. 205-215, 2009.

[43] J. Vandermeer, W. Beamish, T. Milford et al., "iPad-presented social stories for young children with autism," Developmental Neurorehabilitation, vol. 18, no. 2, pp. 75-81, 2015. 